\title{
Neurons in the lateral part of the lumbar spinal cord show distinct novel axon trajectories and are excited by short propriospinal ascending inputs
}

\section{Zs. Antal • L. L. Luz • B. V. Safronov ・ M. Antal • Peter Szucs}

Originally published in Brain Struct Funct (2016) 221:2343-2360. DOI 10.1007/s00429-015-1046-3

Erratum to: Brain Struct Funct DOI 10.1007/s00429-015-1046-3

Unfortunately, Table 1 and the legend have been incorrectly published in the original publication. The correct version is given below. 
Table 1 Position, somatodendritic type and axon trajectory of the recovered neurons

\begin{tabular}{|c|c|c|c|c|c|c|}
\hline \multirow[b]{2}{*}{ cell No } & \multirow[b]{2}{*}{ position } & \multirow[b]{2}{*}{$\begin{array}{l}\text { soma / dendrite } \\
\text { (Lima and Coimbra, } \\
\text { 1986) }\end{array}$} & \multicolumn{3}{|c|}{ axon } & \multirow[b]{2}{*}{ 3-D } \\
\hline & & & $\begin{array}{l}\text { midline } \\
\text { crossing }\end{array}$ & ascending & $\begin{array}{l}\text { ipsilateral } \\
\text { collaterals }\end{array}$ & \\
\hline 1 & L-I & flattened & $\mathrm{AC}$ & c-ALT & DCT & \\
\hline 2 & L-I & fusiform & $\mathrm{AC}$ & c-ALT & MCT & \\
\hline 3 & L-I & flattened & $\mathrm{AC}$ & c-ALT & - & \\
\hline 4 & L-I & flattened & $\mathrm{AC}$ & c-ALT & LCT & \\
\hline 5 & L-I & Flattened & - & - & LCN & \\
\hline 6 & OUT & NA & $\mathrm{AC}$ & c-ALT & - & \\
\hline 7 & OUT & pyramidal & $\mathrm{AC}$ & c-ALT & MCT & \\
\hline 8 & OUT & NA & PC-AC & $\mathrm{i}-\mathrm{ALT}$ & L-III/IV, L-X & + \\
\hline 9 & OUT & NA & PC & c-ALT & L-IV & + \\
\hline 10 & OUT & multipolar & - & - & LCN & + \\
\hline 11 & OUT & NA & $\mathrm{AC}$ & c-ALT & MCT & \\
\hline 12 & OUT & NA & $\mathrm{AC}$ & c-ALT & DCT & \\
\hline 13 & OUT & NA & $\mathrm{AC}$ & c-ALT & - & \\
\hline 14 & OUT & flattened & $\mathrm{AC}$ & c-ALT & LCT & \\
\hline 15 & OUT & NA & PC & C-ALT & - & \\
\hline 16 & OUT & NA & AC & $\begin{array}{l}\text { c-ALT, i- } \\
\text { DLF }\end{array}$ & $\begin{array}{c}\text { DLF-caudal, L-I, } \\
\text { L-III/IV }\end{array}$ & + \\
\hline 17 & OUT & NA & $\mathrm{AC}$ & $\begin{array}{l}\text { c-ALT, i- } \\
\text { DLF }\end{array}$ & Lissauer-tract & + \\
\hline 18 & OUT & NA & - & - & LCN & \\
\hline 19 & OUT & NA & PC-AC & i-ALT & $\mathrm{L}-\mathrm{III} / \mathrm{IV}$ & + \\
\hline 20 & OUT & NA & $\mathrm{PC}-\mathrm{AC}$ & i-ALT & L-III/IV, L-X, L-VII & + \\
\hline 21 & OUT & $\mathrm{NA}$ & PC & C-ALT & L-IIII & + \\
\hline 22 & OUT & NA & PC & c-ALT & L-IIIIV*, L-X* & + \\
\hline 23 & OUT & NA & $\mathrm{AC}$ & c-ALT & MCT & \\
\hline 24 & LSN & NA & PC & c-ALT & DLF, L-X & + \\
\hline 25 & LSN & NA & $\mathrm{AC}$ & $\begin{array}{c}\text { c-ALT, i- } \\
\text { DF }\end{array}$ & DLF-caudal & + \\
\hline 26 & LSN & NA & $\mathrm{AC}$ & $\begin{array}{c}\text { c-ALT, i- } \\
\text { DF }\end{array}$ & DLF-caudal & + \\
\hline 27 & LSN & NA & PC-AC & i-ALT & - & + \\
\hline 28 & LSN & NA & PC & C-ALT & L-VII & + \\
\hline 29 & LSN & NA & - & - & LCN & \\
\hline 30 & LSN & NA & PC & C-ALT & L-V/VI, L-X & + \\
\hline 31 & LSN & NA & $\mathrm{AC}$ & c-ALT & MCT & \\
\hline 32 & LSN & NA & PC-AC & i-ALT & L-V/VI & + \\
\hline
\end{tabular}

Blue values indicate neurons with a projection axon crossing in the posterior commissure and ascending in the cALT. Red values indicate double crossing, i-ALT ascending neurons. Green values indicate neurons with bilateral (ipsi and contra) ascending axon

$L-I$ lamina I, OUT between the lateral edge of the dorsal grey and the LSN, LSN lateral spinal nucleus, NA not applicable, $A C$ anterior commissure, $P C$ posterior commissure, $c-A L T$ contralateral anterolateral tract, $i-A L T$ ipsilateral anterolateral tract, $D L F$ dorsolateral funiculus, $D F$ dorsal funiculus, $L-I-X$ lamina I-X, 3-D neuron reconstructed in three-dimensions with Neurolucida, $D C T$ dorsal collateral type projection neuron (see Szucs et al. 2010), MCT mixed collateral type projection neuron (see Szucs et al. 2010), LCT lateral collateral type projection neuron (see Szucs et al. 2010) 